\title{
ESTERILIZACIÓN QUIRÚRG ICA POR VÍA TRANSUMBILICAL CON ANESTESIA LOCAL EN PUÉRPERAS RECIENTES
}

\author{
Luis Ignacio de la Torre Ch. ${ }^{1}$, Erasmo Bravo O. ${ }^{1}$, Harald Riesle P. ${ }^{1}$, Verónica Chamy P. ${ }^{1}$ \\ Karina Michea N. ${ }^{a}$ \\ ${ }^{1}$ Servicio de Ginecología y Obstetricia, Hospital Carlos Van Buren, Escuela de Medicina Universidad de Valparaíso.
}

anterna, Escuela de Medicina, Universidad de Valparaíso.

\section{RESUMEN}

Objetivo: Comparar la esterilización quirúrgica vía transumbilical en puérperas recientes con anestesia local versus anestesia regional. Método: Durante el período de estudio comprendido entre septiembre de 2003 a septiembre de 2004, se realizaron en el hospital Carlos Van Buren, 196 ligaduras posteriores a un parto vaginal. El grupo 1 (anestesia local) quedó constituido por 136 mujeres y el grupo 2 (anestesia regional) por 60 mujeres. Resultados: No hubo diferencias significativas entre los grupos analizados de las características obstétricas y antropométricas, tiempo operatorio, percepción del dolor y complicaciones postoperatorias. Hubo una reducción significativa en el período de latencia entre el parto y la salpingoligadura, de 30 horas en el grupo de anestesia local versus 62 horas en el de anestesia raquídea $(p<0,00001)$ y en días de hospitalización de 2,2 versus 3,9 días $(p<0,0001)$, respectivamente. Conclusiones: La esterilización quirúrgica realizada con anestesia local disminuyó de manera significativa el tiempo de latencia entre el parto y la ligadura y los días de hospitalización, permitiendo el retorno precoz de las madres a su ambiente familiar y una disminución de los costos asistenciales.

\section{PALABRAS CLAVES: Esterilización quirúrgica, esterilización transumbilical, puérperas recientes, anestesia local}

\section{SUMMARY}

Objectives: To compare the tubal sterilization trough subumbilical incision performed with local anesthesia versus spinal anesthesia in the postpartum period. Methods: We perform 196 tubal sterilization procedures after a vaginal delivery. The study group 1 (local anesthesia) included 136 women and the study group 2 (spinal anesthesia) 60 women. Results: The study showed no statistical significant difference between the two groups, in the obstetrics and anthropometrics characteristics, neither in the average surgical time, the pain score (measured with the analogue visual scale), or postoperative short term complications. The only statistically significant difference was found in the period of time between the vaginal delivery and the sterilization procedure (30 hours in group 1 and 62 hours in group $2, p<0.0001$ ), and in the hospitalization number of days (2.2 days in group 1 and 3.9 days in group $2, p<0.0001)$. Conclusions: The transumbilical tubal sterilization under local anesthesia in postpartum period lowers the hospital stay and the time between childbirth and the surgical procedure an allowing the mothers an early return to their home, with less cost for the health system.

\section{KEY WORDS: Surgical sterilization, subumbilical incision, postpartum period, local anesthesia}




\section{INTRODUCCIÓN}

La esterilización quirúrgica femenina es en la actualidad una de las cirugías ginecológicas más realizadas en el mundo. En Chile, este procedimiento aumentó desde el 2000, producto del cambio en la legislación que lo regulaba, liberalizando su solicitud y aplicación. Antes del 2000, una mujer podía solicitar la esterilización quirúrgica si reunía los siguientes requisitos: edad mayor de 30 años, tener a lo menos 3 hijos vivos y estar gestando el cuarto, con autorización del marido. Una circunstancia especial la constituían aquellas madres portadoras de alguna patología que contraindicara el embarazo, las que podían ser ligadas sin reunir los criterios nombrados con anterioridad. La legislación aprobada en el año 2000, considera la esterilización como un método para regular la fertilidad y no un acto médico, por lo que se exige solamente el deseo voluntario e informado por parte de la paciente para aprobar su realización.

Este tipo de anticoncepción es permanente, altamente efectiva, muy bien tolerada y segura, con una tasa de embarazos de 1-2 por cada 1000 mujeres intervenidas, ocurriendo en estos casos más frecuentemente embarazos ectópicos. La tasa de falla de la técnica es levemente mayor en aquellas mujeres que se ligan antes de cumplir 30 años (1-7).

La forma más común de realizar la esterilización quirúrgica femenina es la salpingoligadura (SLG), en la cual se liga un segmento de la trompa (generalmente ístmico) para impedir la fecundación. Existen numerosas técnicas descritas, siendo las más utilizadas en la actualidad las descritas por Parkland y Pomeroy. Existen numerosas vías de abordaje para realizar la esterilización quirúrgica femenina, destacando en la actualidad la vía laparoscópica, la mini laparotomía, la transumbilical, la vía vaginal y la histeroscópica, entre otras, cada una con ventajas y desventajas, como también el momento para su aplicación (5-10,11-14).

La mini laparotomía transumbilical es la técnica de elección para las puérperas recientes. La laparoscópica y mini laparotomía transversa suprapúbica son de elección en las mujeres que solicitan SLG en algún periodo de sus vidas sin relación con embarazo (1-4,15). Las complicaciones quirúrgicas ocurren en promedio en un $0,1 \%$ de las intervenciones, pero varían según el tipo de procedimiento. Estas complicaciones incluyen infección, lesión vesical o intestinal, hemorragia interna y las derivadas del tipo de anestesia empleada (8-10,16-18).
El servicio de Ginecología y Obstétrica del Hospital Carlos Van Buren, ha presentado un aumento de solicitudes de SLG, lo que ha determinado que el número de esterilizaciones quirúrgicas haya aumentado en un $100 \%$ en los últimos 9 años (301 en 1997 a 612 el 2004), lo que ha generado la necesidad de destinar mayor número de horas quirúrgicas y anestésicas.

Uno de los grupos mayoritarios que solicitan SLG, son las puérperas de parto vaginal (11). Para este grupo en particular se recomienda realizar el procedimiento quirúrgico en las primeras 24 a 72 horas posparto, posterior a lo cual se describen mayores dificultades técnicas intra y postoperatorias, siendo el límite recomendado en la actualidad 5 a 6 días post parto $(15,16,19)$.

En septiembre de 2003, iniciamos un estudio descriptivo de la latencia de la SLG en las puérperas de parto vaginal de nuestro servicio, observando un promedio de 95 horas entre el parto y la ligadura, con un mínimo de 12 y un máximo de 105 horas. Esta situación determinaba en algunos casos que las pacientes debieran diferir el procedimiento, lo que se tradujo en una mayor espera de las puérperas para ser dadas de alta, en aumento de la lista de espera para esa cirugía y en la ocurrencia de embarazos no deseados, en aquellas pacientes que se retiraban del hospital sin esperar el procedimiento.

La razón de esta latencia radicaba en la falta de horas anestésicas para efectuar los procedimientos, situación que no podría ser revertida hasta mediados del 2006. Por esta razón realizamos una búsqueda de literatura científica que aportara experiencias con técnicas de SLG en puérperas recientes con anestesia local. Los trabajos encontrados fueron numerosos y la evidencia era sólida en demostrar que no habían mayores riesgos que en las técnicas con anestesia regional (20-25).

Establecimos como hipótesis de trabajo que la salpingoligadura transumbilical realizada con anestesia local, tiene una latencia menor posparto que la realizada con anestesia regional, sin modificar significativamente el tiempo operatorio, evaluando las complicaciones intra y postoperatorias y la percepción del dolor postoperatorio utilizando la escala visual análoga.

\section{MATERIAL Y MÉTODO}

Desde el 25 de septiembre de 2003 al 25 de septiembre de 2004 se realizó un protocolo de SLG en puérperas recientes de parto vaginal atendidas en el Servicio de Ginecología y Obstetricia 
del Hospital Carlos Van Buren. Durante este período hubo 3231 partos y 491 ligaduras, de las cuales 196 fueron posteriores a un parto vaginal.

A todas las puérperas que habían solicitado SLG, se les informó la posibilidad de realizar la cirugía con anestesia raquídea o local, según la disponibilidad de anestesista del momento. Se solicitó consentimiento informado para la cirugía.

La asignación del tipo de anestesia utilizado para la intervención no fue randomizada, sino por conveniencia, dependiendo de la disponibilidad de anestesista al momento de la cirugía. Hubo una relación 2 a 1, a favor de la ligadura con anestesia local. El grupo 1 (anestesia local) quedó constituido por 136 mujeres y el grupo 2 (anestesia regional) por 60 mujeres. Se estableció un protocolo anestésico único para cada grupo e igual técnica quirúrgica para ambos.

Se consignaron los antecedentes obstétricos (gestaciones, paridad y abortos), antropométricos (peso, talla e IMC), fecha y hora del parto, tiempo de latencia entre el parto y la ligadura expresado en horas, duración de la intervención en minutos, conversión de anestesia local a raquídea, complicaciones intraoperatorias, percepción del dolor postoperatorio con la escala visual análoga (EVA), días de hospitalización post parto.

Análisis estadístico: Los datos obtenidos fueron analizados utilizando paquete estadístico Stata 8 , con análisis descriptivo y estadística inferencial con prueba de chi cuadrado para variables categóricas y $\mathrm{t}$ de student para variables continuas.

Protocolo anestesia local: Una vez preparada la paciente para la cirugía y estando monitorizada en pabellón, se administran por vía intravenosa $1,5 \mu \mathrm{g} / \mathrm{k}$ de fentanilo, más $2 \mathrm{mg}$ de midazolam y $0,4 \mathrm{mg}$ de atropina. Luego se infiltra la zona periumbilical con $20 \mathrm{ml}$ de lidocaína al $2 \%$, alcanzando la aponeurosis.

Protocolo anestesia regional: Las pacientes recibieron anestesia regional según la técnica clásica utilizando 7,5 mg de bupivacaína al 0,5\% y $20 \mu \mathrm{g}$ de fentanilo. En aquellas pacientes de talla menor a $150 \mathrm{~cm}$ la dosis de bupivacaína fue menor.

Técnica quirúrgica: Laparotomía mínima transumbilical. Identificación de ambas trompas, salpingoligadura en porción ístmica según técnica de Parkland, con catgut simple 0 , revisión de hemostasia y recuento de gasas, cierre de aponeurosis con gareta de Polysorb 1, compresión periumbilical.

Analgesia: Para ambos grupos se consideró la utilización intraoperatoria de $2 \mathrm{~g}$ de metamizol parenteral; en casos de alergia reportada se administró previo a la cirugía ketoprofeno 100 mg IM.

\section{RESULTADOS}

Durante el período de estudio se atendieron 3231 partos y se realizaron 496 ligaduras, de las cuales 300 se realizaron durante la cesárea y 196 posterior a un parto vaginal. El grupo de estudio está constituido por las 196 pacientes que fueron ligadas tras un parto vaginal (Tabla I).

Ambos grupos son similares en las variables que se relacionan con un mayor o menor éxito del procedimiento. Con respecto a las variables postoperatorias no hubo diferencias significativas entre los grupos respecto al tiempo operatorio y las complicaciones intraoperatorias, ni en la percepción del dolor postoperatorio medido por EVA; hubo diferencias significativas al analizar la latencia en horas entre el parto y la SLG (Tabla II).

Durante el desarrollo del protocolo y debido a la característica de centro docente asistencial de nuestro servicio, la cirugía fue desarrollada por ginecólogos y becados (Tabla III, IV). Al analizar las variables intra y postoperatorias de las cirugías realizadas por ginecólogos y becados, es posible observar que el único aspecto que presenta diferencias significativas entre los dos grupos fue la latencia entre el parto y la ligadura, fenómeno atribuible a que la mayoría de los procedimientos realizados con anestesia espinal fueron realizados por ginecólogos y la participación de los becados se concentró en el grupo de anestesia local.

Con respecto a las conversiones de anestesia local a espinal, se presentaron 5 casos, los cuales se concentraron en los 3 primeros meses de implementada la técnica y se relacionaron princi-

Tabla I

\section{CARACTERÍSTICAS OBSTÉTRICAS Y ANTROPOMÉTRICAS DE LAS PACIENTES DEL GRUPO DE ESTUDIO}

\begin{tabular}{lccl}
\hline & $\begin{array}{c}\text { Anestesia } \\
\text { local }\end{array}$ & $\begin{array}{c}\text { Anestesia } \\
\text { regional }\end{array}$ & $\begin{array}{l}\text { Valor } \\
p\end{array}$ \\
\hline Número de pacientes & 136 & 60 & - \\
Edad & 32,4 & 31,7 & $0,39^{* *}$ \\
Gestaciones* & 4 & 3,6 & $0,09^{* *}$ \\
Paridad* $_{\text {Abortos* }}^{*}$ & 3,6 & 3,3 & $0,16^{* *}$ \\
Peso* (kilos) $_{\text {Talla* (cm) }}$ & 0,5 & 0,25 & $0,1^{* *}$ \\
IMC & 73,2 & 74,0 & $0,9^{* *}$ \\
\hline
\end{tabular}

*Valor promedio. **Diferencia no significativa. 
Tabla II

CARACTERÍSTICAS INTRA Y POST OPERATORIAS DE LOS GRUPOS ESTUDIADOS

\begin{tabular}{lccc}
\hline & Anestesia local & Anestesia raquídea & Valor $p$ \\
\hline Duración promedio de la intervención (minutos) & 22,9 & 24,2 & $0,44^{*}$ \\
Percepción del dolor postoperatorio (EVA) & 2,8 & 2,6 & $0,12^{*}$ \\
Complicaciones intraoperatorias & 0 & 0 & - \\
Latencia promedio parto-ligadura (minutos) & 30 & 62 & $<0,00001^{* *}$ \\
\hline
\end{tabular}

*Diferencia no significativa. ${ }^{* *}$ Diferencia significativa.

palmente con una mayor latencia entre el parto y la ligadura y un IMC mayor a 30, pero la diferencia de ambos aspectos no fue estadísticamente significativa (Tabla V).

\section{DISCUSIÓN}

Este estudio permite afirmar que la anestesia local es tan adecuada como la anestesia regional para realizar la esterilización quirúrgica en puérperas recientes por vía transumbilical, sin aumentar el tiempo operatorio, la percepción de dolor postoperatorio, ni las complicaciones intraoperatorias. Esto permitió disminuir significativamente la latencia entre el parto y la ligadura, reduciendo los días de hospitalización y los costos asociados de forma directa o indirecta al procedimiento. Se demostró que esta técnica es altamente reproducible y fácil de aprender, lo cual queda respaldado por varios trabajos citados en las referencias, dos de los cuales son de hospitales chilenos $(22,25)$, los que han demostrado resultados similares. Además la realización de la cirugía por becados o cirujano no modificó significativamente los tiempos quirúrgicos ni las complicaciones.

Al analizar las causales de los casos en que se produjeron conversiones anestésicas, resulta evidente que la indicación de la vía quirúrgica y el tipo de anestesia, debe considerar aspectos como el tiempo de latencia entre el parto y la ligadura y el IMC de las pacientes, los cuales si bien no alcanzaron diferencias estadísticamente significativas, entre las pacientes del grupo de anestesia local que no requirieron conversión y las que si

Tabla III

\section{CALIDAD DEL MÉDICO QUE REALIZÓ LA CIRUGÍA}

\begin{tabular}{llc}
\hline & Anestesia local & Anestesia raquídea \\
\hline Ginecólogos & 112 pacientes (83\%) & 56 pacientes (93\%) \\
Becados & 24 pacientes (27\%) & 4 pacientes (7\%)
\end{tabular}

Tabla IV

\section{VARIABLES INTRA Y POSTOPERATORIAS SEGÚN LA CALIDAD DEL MÉDICO QUE REALIZÓ EL PROCEDIMIENTO}

\begin{tabular}{lccc}
\hline & $\begin{array}{c}\text { Ginecó- } \\
\text { logos }\end{array}$ & $\begin{array}{c}\text { Beca- } \\
\text { dos }\end{array}$ & $\begin{array}{c}\text { Valor } \\
p\end{array}$ \\
\hline Número pacientes & 168 & 28 & - \\
Tiempo operatorio (minutos) & 23 & 25 & $0,3^{*}$ \\
Dolor postoperatorio & 2,5 & 2,8 & $0,09^{*}$ \\
Complicaciones postoperatorias & 0 & 0 & - \\
Latencia parto ligadura (horas) & 48 & 14 & $<0,00001^{* *}$ \\
\hline
\end{tabular}

*Diferencia no significativa.

${ }^{* *}$ Diferencia significativa.

requirieron, son un factor técnicamente atribuible al fracaso de la técnica anestésica por presentar variables técnicas y farmacocinéticas que pueden afectar el resultado.

El impacto económico de la implementación de esta técnica en nuestro servicio fue significativo, pero sin duda el retorno precoz de las pacientes a su ambiente familiar fue el mayor beneficio de esta medida y la principal razón de su implementación.

En un servicio público, donde las necesidades siempre superan los recursos disponibles y donde la capacidad de adquirir materiales o insumos es muy limitada, el deseo de cambiar aspectos que parecen inherentes al mismo puede dar lugar al desarrollo y aplicación de técnicas importadas de

Tabla V

CARACTERÍSTICAS DE LAS PACIENTES QUE REQUIRIERON CONVERSIÓN ANESTÉSICA

\begin{tabular}{lccc}
\hline & Convertidas & No convertidas & Valor $p$ \\
\hline IMC & 32,1 & 29,8 & $0,2^{*}$ \\
Latencia (horas) & 30,1 & 38,2 & $0,6^{*}$ \\
\hline
\end{tabular}

*Diferencia no significativa. 
otros servicios, lo que puede permitir cambios significativos en nuestro ámbito hospitalario mejorando la gestión clínica. En la actualidad existen variadas alternativas que nos hubiesen permitido cumplir en mayor o menor grado con el paradigma inicial de nuestro trabajo, proveer de un método simple, rápido, mínimamente invasivo, ambulatorio, económico, fácil de aprender, con pocas o ninguna complicación postoperatoria y que no requiere de anestesia regional o general, siendo las técnicas histeroscópicas un buen ejemplo de esto, aunque para nosotros es una alternativa distante, debido al costo de los instrumentos y los insumos, pero debe ser materia de análisis para futuras adquisiciones debido a la excelente evidencia que respalda su uso $(14,21)$.

\section{CONCLUSIONES}

La esterilización quirúrgica por vía transumbilical con anestesia local en puérperas recientes, comparada con la realización del mismo procedimiento bajo anestesia regional, permitió menores gastos en insumos y horas anestésicas, disminución del tiempo de hospitalización, retorno precoz de las madres al ambiente familiar, menor costo directo e indirecto de la técnica.

\section{BIBLIOGRAFÍA}

1. Magos A, Chapman L. Hysteroscopic tubal sterilization. Obstet Gynecol Clin North Am 2004;31:705-19.

2. Allyn DP. Presterilization counseling and women's regret about having been sterilized. J Reprod Med 1986;31:1027-32.

3. Grubb GS, Peterson HB, Layde PM, Rubin GL. Regret alters decision to have a tubal sterilization. Fertil Steril 1985;44:248-53.

4. Wilcox LS, Chu SY, Eaker ED, et al. Risk factors for regret after tubal sterilization: 5 years of follow up in a prospective study. Fertil Steril 1991;55:927-33.

5. Tulandi T. Tubal sterilization. N Eng J Med 1997;336: 796-7.

6. Shaw R, Russel I, Settatree R, et al. Male and female sterilization. Evidence-Bases guidelines No. 4. London: Royal College of obstetricians and Gynaecologist; 1999.

7. Varma R. Failed sterilization: evidence-based review and medico legal ramifications. BJOG 2004;111(12): 1322-32.

8. American College of Obstetrics and Gynecology.
Sterilization ACOG technical bulletin No. 222. ACOG, Washington, DC, 1996.

9. American College of Obstetrics and Gynecology. ACOG practice bulletin No. 46, September 2003. Int J Gynaecol Obstet 83(3):339-50.

10. Peterson HB, Xia Z, Hughes JM, et al. The risk of pregnancy after tubal sterilization: findings from the US Collaborative Review of Sterilization. Am J Obstet Gynecol 1996;174:1161-8.

11. Skajaa K. Sterilization of women in the puerperium. Acta Obstet Gynecol Scand 1932;12:114.

12. Wortman J. Tubal sterilization: Review of methods. Popul Rep C 1976;7:73-96.

13. da Costa Leite I. Female sterilization in Latin America: cross national perspectives. J Biosoc Sci 2004; 36(6):683-98.

14. Rosen, DM. Learning curve for hysteroscopic sterilisation: lessons from the first 80 cases. Aust $\mathrm{N} \mathrm{Z} \mathrm{J}$ Obstet Gynaecol feb 2004; 44(1):62-4

15. Curtis, KM. Regret following female sterilization at young age: a systematic review. Contraception feb 2006; 73(2):205-10.

16. Peterson HB, Xia Z, Hughes JM, et al. The risk of ectopic pregnancy after tubal sterilization. N Eng J Med 1997;336:762-7.

17. Miesfeld RR, Giarratano RC, Moyers TG. Vaginal Tubal Ligation-is infection a significant risk? Am J Obstet Gynecol 1980;137:183-8.

18. Bhiwandiwala PP, Mumford SD, Feldblum PJ. Menstrual pattern changes following laparoscopic sterilization with different occlusion techniques: a review of 10004 cases. Am J Obstet Gynecol 1983;145:68494.

19. Potter JE. Frustrated demand for postpartum sterilization in Brazil. Contraception 2003;75(5):385-90.

20. Crighton IM, Hobbs GJ, Wrench IJ. Analgesia after day case laparoscopic sterilization: a comparison of tramadol with paracetamol and paracetamol/codeine combinations. Anesthesia 1997;52(7):649-52.

21. Mazdisnian F, Palmieri A, Hakakha B, et al. Office microlaparoscopy for female sterilization under local anesthesia. A cost and clinical analysis. J Reprod Med 2002;47(2):97-100.

22. Perucca E, Iracheta A, Siebert A, et al. Experiencia clínica minilap para la esterilización quirúrgica. REV ChiL OBSTET GINECOL 2002;67(4):305-8.

23. Uchida H. Uchida tubal sterilization. Am J Obstet Gynecol 1975;121:153.

24. Nisanian A. Outpatient minilaparotomy sterilization with local anestesia. J Reprod Med 1990;35(4):3803.

25. Grudsky C, Valdivia I, Herrera M, et al. Tubal ligation by mini laparotomia. Rev CHIL OBStet GineCOL 1981; 46(4):198-205. 